\title{
Die Kompetenzverteilung in der Deutschen Reichsverfassung 1871
}

\author{
The Distribution of Competences in Bismarck's Constitution 1871
}

Bismarck's Constitution is the basis of the most important example of a living monarchical federal state. In it, the monarchical position, even in the constituent states, is clearly more emphasized than in its Frankfurt predecessor of 1849 and left untouched in the text until 1918. On the other hand, however, this position is increasingly undermined by a development which, to the detriment of the constituent states, makes use of farreaching competences - as under the American Constitution - and, moreover, permits amendments and breaches of the constitution by simple majorities.

Keywords: constitution as simultaneous diplomatic paper - contrasts between textual constitution and realities monarchical instead of republican federation

\section{Zum Kreis der Vorprägungen}

Bekanntlich gehört es zur positivistischen Reduktion des seinerzeit führenden reichsdeutschen Öffentlichrechtlers Paul Laband, in seiner magistralen Behandlung der hier thematisierten Verfassung verfassungshistorische Hintergründe ausgeblendet zu haben. ${ }^{1}$ Deshalb sei zu Beginn der hiesigen Ausführungen auf gewisse kompetenzrelevante Vorgegebenheiten aufmerksam gemacht, und zwar unbeschadet des von maßgeblicher parlamentarischer Seite damals als „völlig originell“ ${ }^{\text {” }}$ gewürdigten und später dann im Wesentlichen adaptierten Gubernativentwurfs der deutschen Verfassung von 1867/71. Herrschte doch damals allenthalben das Bewusstsein vor, die primär staatenbündische Konstruktion des Deutschen Bundes, eines laut

\footnotetext{
${ }^{1}$ Grundlegend die zeitgenössische Kritik von GIERKE, Staatsrecht.

${ }^{2}$ So der nationalliberale Abgeordnete Johannes von Miquel, siehe HoltzendorfF, Bezold, Materialien 1, 106 (9. Sitz., 9. 3. 1867).
}

Wiener Schlussakte völkerrechtlichen Vereins, ${ }^{3}$ staatsrechtlich i.S. bundesstaatlicher Verdichtung umzuwandeln. ${ }^{4}$

Erläuternd sei dazu, wenn man aus dem deutschsprachigen Nordwesten kommt, nur zu gern der frühe Bundesstaatstheoretiker und kurhannoversche Staatsmann Ludolf Hugo herangezogen, dessen Propagierung des „duplex regimen" in seiner alsbald in sieben Auflagen erschienenen Helmstedter Dissertationsschrift ${ }^{5}$ be-

\footnotetext{
${ }^{3}$ Art. 1 WSA (1820), HubER, Dokumente 1, Nr. 31.

${ }^{4}$ Vgl. nur die parlamentarischen Verfassungsdebatten von 1867-71 im von BEZOLD hrsg. Registerbd. (HoLTZENDORFF, BEZOLD, Materialien 47f.), in dem „Bundesstaat" 28-mal und "Bundesstaat oder Staatenbund“ 23-mal ausgewiesen sind.

${ }^{5}$ Siehe seine lateinisch geschriebene Dissertation „De Statu Regionum Germaniae", erschienen erstmals 1661, um dann bis 1736 insges. sieben Auflagen zu erfahren; die 8. Auflage erschien dann, übersetzt von PFANNENSCHMID, erstmals 2005 auf Deutsch. Dabei beruhte die seinerzeitige Auflagenhäufigkeit darauf, dass die Arbeit praktikable Vorschläge für die anhaltende Diskussion über die Verfassung des Alten
} 
trächtliche Nachwirkungen für die Ausbildung des modernen Bundesstaats hatte - sieht diese Schrift doch über die Sprengung des Bodinschen Souveränitätsmonismus hinaus weit weniger geläufig zugleich eine konkrete Kompetenzverteilung zwischen dem Alten Reich und seinen Gliedern vor, ${ }^{6}$ was in bewundernswerter Hellsichtigkeit nach wie vor als Grundlage bundesstaatlicher Zuständigkeitsverteilung gelten kann.

Indessen ist das Crescendo dieses Auftakts für unser Thema gleich zu diminuieren. Denn die Wirkungen dieser Schrift kommen im 19. Jahrhundert nur noch indirekt zum Zuge, und zwar maßgeblich durch die Mittlerrolle der Verfassung der Vereinigten Staaten und daran anschließend durch die der Paulskirche. ${ }^{7}$ Für die Reichsverfassung von 1871 stehen ihr norddeutsches Vorstück von 1867 im Vordergrund und in geringerem Maße noch schweizerische Erfahrungen. ${ }^{8}$

Letztere Einflussansage gilt es freilich zu differenzieren. Die nach dem dortigen Sonderbundskrieg geschaffene Schweizer Bundesverfassung von 1848 galt 1867/71 wegen ihrer bundesseitigen Zuständigkeitsschwächen als wenig attraktiv und wurde ihrerseits 1874, umgekehrt auch unter dem Eindruck der Bismarckschen Reichsverfassung (BRV), einer Totalrevision unterzogen. ${ }^{9}$ Und was die amerikanische Verfassung be-

Reichs bot, deren Mängelbehebung der Westfälische Frieden auf den nächsten - dann immerwährenden Reichstag verschoben hatte.

${ }^{6}$ Dazu SchenNaCH, Kompetenzverteilung u. eingehend PfANNENSCHMID, Ludolf Hugo 134ff. m.w.N. und tabellarischer Übersicht zur Kompetenzverteilung 150.

${ }^{7}$ Amerikanische Verfassung vom 17.9.1787; FRV vom 28. 3. 1849.

${ }^{8}$ Verfassung des Norddeutschen Bundes vom 16. 4. 1867 sowie die schweizerische Verfassung vom 12. 9. 1848, zu Letzterer eingehend RAPPARD, Bundesverfassung $121 \mathrm{ff}$.

${ }^{9}$ Revidierte Verfassung vom 29.5.1874, dazu ebd. $315 \mathrm{ff}$. u. der Beitrag von Schindler in diesem Band. trifft, so stand man ihr im kleindeutschen Raum wegen der bundesstaatlichen Größenverhältnisse tendenziell näher. Indessen hatte ihr Ansehen durch den erst wenige Jahre zuvor beendeten Bürgerkrieg Federn gelassen - einschließlich der dafür mitverantwortlichen Rolle ihrer Verfassungsgerichtsbarkeit. ${ }^{10}$

Demgegenüber stand nun für Kompetenzfragen eine innerdeutsche Diskussion im Vordergrund, die von der Frankfurter Reichsverfassung der Paulskirche ausging. Dazu sei generell auf die juristisch bislang zu wenig beachtete umfangreiche Föderalismusstudie des Neuhistorikers Michael Dreyer ${ }^{11}$ verwiesen und eine zweifache Reaktion auf die Frankfurter Reichsverfassung (FRV) hervorgehoben: zum einen normativ in weniger unitarischer denn stärker einzelmonarchischer Absicht die Erfurter Unionsverfassung von $1850^{12}$ sowie bundesstaatstheoretisch die die doppelte Souveränität bis an den Rand staatlicher Spaltung steigernde Lehre des Historikers Georg Waitz, ${ }^{13}$ der damit als einer der maßgeblichen Verfassungsschöpfer der Paulskirche 1852 ex post deren Kardinalfehler beheben zu können meinte. Doch wurde die FRV mit ihrer ausgefeilten Kompetenzverteilung gerade auch $\mathrm{zu}$ Beginn der sog. Neuen Ära in Preußen, d.h. zu Beginn der 1860er-Jahre Fuß- und Angelpunkt für alle Reformüberlegungen bleiben, den Deutschen Bund zum Bundesstaat zu verdichten.

\footnotetext{
${ }^{10}$ Das oberste Gericht hatte nämlich in folgenschwerer Weise mit seiner berühmt-berüchtigten Dred Scott-Entscheidung vom 1857 zur Entstehung des amerikanischen Bürgerkriegs beigetragen; näher LOEWENSTEIN, Verfassungsrecht 578f.

${ }^{11}$ DREYER, Föderalismus, insbes. 160ff.

12 Vom 28. 5. 1850, abgedruckt bei HubER, Dokumente 1, Nr. 209, 177.

${ }^{13}$ Waitz veröffentlichte seine Theorie erst 1852, dazu genauer DREYER, Föderalismus 205, Fn. 135, wobei es ihm darum ging, die Fehler der Paulskirche zu vermeiden, s.a. ebd. 209 Fn. 148 und 211; weiter WIEDERIN, Bundesrecht und Landesrecht 7f., der zu Recht den Verzicht dieser Theorie auf Konfliktlösung kritisiert.
} 
Als Hochphase dafür sind fraglos die Jahre ab $1861 \mathrm{zu}$ nennen. Angeführt seien hierzu etwa die österreichischen und preußischen Bundesreformvorschläge und die Abgeordnetentage von Weimar und Frankfurt a.M. ${ }^{14}$ Überdies nicht zu vergessen sind aus dem gesellschaftlichen Bereich der kleindeutsche Nationalverein mit seinem Verlangen einer Einführung der FRV sowie spürbar zurückhaltender der großdeutsche Reformverein,15 der mit Rücksicht auf Österreich für eine weniger vorgespurte Weiterentwicklung der Deutschen Bundesakte eintrat. Letzterer bietet mit seiner Erklärung zur angestrebten Kompetenzaufteilung immerhin eine gruppierende Zusammenfassung, der als oberbegriffliche Kompetenztrias die Zukunft gehören sollte, wird es doch als „ein dringendes und unabweisliches Bedürfnis [bezeichnet], sowohl [...] die Machtstellung nach außen als [auch] die Wohlfahrt und bürgerliche Freiheit im Innern kräftiger als bisher zu fördern". ${ }^{16}$

\section{Bundesstaatliche Reichsgründung durch Kompetenzmimikry}

Der eben erwähnten Kompetenztrias sollten die vornehmlich in Art. 4 BRV katalogisierten Reichszuständigkeiten durchaus entsprechen. Die äußere Machtstellung wird konkretisiert durch Zuständigkeiten für: Kolonisation, Schutz des deutschen Handels im Ausland einschließ-

\footnotetext{
14 Österreichische Denkschrift vom 31. 7. 1863, HUBER, Dokumente 2, Nr. 103, 107; preuß. Antwortschreiben vom 22. 9. 1863, ebd. Nr. 110. Zu den Abgeordnetentagen in Weimar vom 28. 9. 1862, ebd. Nr. 101, 100, in Frankfurt a.M. vom 21./22. 8. 1863, ebd. Nr. 106, 105. ${ }^{15}$ Resolution des Nationalvereins vom 16.10.1862, HubER, Dokumente 2, Nr. 102, 101; Programm des Deutschen Reformvereins vom 28.10.1862, ebd. Nr. 90, 89.

${ }^{16}$ Vgl. Programm des Reformvereins, HubER, Dokumente 2, Nr. 90, 89.
}

lich der deutschen Überseeschifffahrt samt konsularischer Vertretung sowie nicht zuletzt für Kriegsmarine und Heerwesen. ${ }^{17}$

Und der Sicherheit wie Wohlfahrt dienen: das übergreifende Verkehrswesen, womit es im Einzelnen um Eisenbahnen, Straßen, Binnenschifffahrt und - modern gesprochen - Telekommunikation geht. Dazu kommen die früheren Zollvereinsanliegen der Vereinheitlichung von Zöllen, Maßen, Münzen, Gewichten samt allgemeinen Bestimmungen über das Bankwesen ${ }^{18}$ und weiter die alsbald breit genutzte Befugnis zur Schaffung von Nationalgesetzbüchern für das Zivil- wie Strafrecht sowie Gerichtsverfahren. ${ }^{19}$

Bleibt als dritter Materienbereich noch die grundrechtserinnernde Dimension bürgerlicher Freiheit durch Regelung des Indigenats, der persönlichen und wirtschaftlichen Freizügigkeit samt Schutz des geistigen Eigentums sowie der Kompetenz zur Gestaltung des Presse- und Vereinswesens. ${ }^{20}$

Die Etablierung dieser Reichszuständigkeiten war freilich mit Schwierigkeiten verbunden, die im Blick auf ihre spätere Nutzung nicht übergangen werden dürfen. Zum einen war es die für jede Bundesstaatsgründung unausweichliche Konsequenz, die das vormalige Mitglied des großdeutschen Reformvereins, der nunmehrige württembergische Premier Hermann Freiherr von Mittnacht 1870 in der Stuttgarter Kammer der Abgeordneten den Gegnern der Beitrittsvereinbarung zur kleindeutschen BRV entgegenhielt. $^{21}$ Es könne keinen Bundesstaat geben, in welchem nicht die einzelnen Glieder Teile ihrer vollen Souveränität auf den Gesamtstaat über-

${ }^{17}$ In der genannten Reihenfolge: Art. 4 Nr. 1, 7, 14 i.V.m. Art. 56, 53 und 57ff. BRV.

${ }^{18}$ In der genannten Reihenfolge: Art. 4, 7-9 und Nr. 3f. BRV.

${ }^{19}$ In der genannten Reihenfolge: Art. 4 Nr. 13, 11 BRV. ${ }^{20}$ In der genannten Reihenfolge: Art. 3, 4 Nr. 1, 6 und $16 \mathrm{BRV}$.

${ }^{21}$ So HoltzendorfF, Bezold, Materialien 3, 541 (5. Sitz., 23. 12. 1870). 
trügen. Sie erlangten dabei Ersatz in ihrer Stellung zur und ihrer Teilnahme an der Gesamtheit. Und zum anderen trat in klarer Abweichung von den Vereinigten Staaten und der Schweiz eine damals spezifisch deutsche Gegebenheit hinzu: die Konstruktion eines monarchischen Bundesstaats.

Diese Besonderheit ist schärfer auszuleuchten, nicht zuletzt auch als Beispiel diplomatischer Kunst Otto von Bismarcks. So schreibt er hinsichtlich seines Verfassungsentwurfs in den Putbuser Diktaten ${ }^{22}$ an seine in Berlin verbliebenen Mitarbeiter: „Man wird sich in der Form mehr an den [überkommenen] Staatenbund halten müssen, diesem aber praktisch die Natur des Bundesstaats geben mit elastischen, unscheinbaren, aber weitgreifenden Ausdrücken. ${ }^{23}$ Hiermit wurde die Grundlage für eine Verfassungsund namentlich Kompetenzausdehnung geschaffen, die Dynamik statt Statik anzeigte und sich mit dem späteren Topos der "Wanderung zur Zentrale" bzw. zum Zentralstaat überschreiben lässt. Die bemerkenswerte Parallele zur amerikanischen Verfassung und ihrer verfassungsgerichtlich unterstützten Kompetenzextension, die mit dem Zwillingsgeschütz von "sweeping clause“" und „implied powers" arbeitete, ${ }^{24}$ wird auch noch später auffallen.

Doch seien zunächst die spezifischen Zusatzschwierigkeiten konkretisiert, die sich bei der Schaffung eines monarchischen Bundesstaats gegenüber der einer republikanischen Föderati-

\footnotetext{
${ }^{22} \mathrm{Zu}$ diesen im Herbst 1866 auf Rügen entstandenen Niederlegungen eingehend BECKER, Bismarcks Ringen 236ff. und zuvor von staatsrechtlicher Seite KAUFMANN, Bismarcks Erbe 161ff. auf den Kontrast zum republikanischen Bundesstaat eingehend, weiter s.u. Fn. 33.

${ }^{23}$ Zit. n. BECKER, Bismarcks Ringen 240; s.a. KAUFMANN, Bismarcks Erbe 170f., der darauf aufmerksam macht, dass Bismarck hierbei die Erhebung von Verfassungsrechtsfragen durch politische Mehrheitsentscheidungen vermieden wissen wollte.

${ }^{24}$ Dazu näher LOEWENSTEIN, Verfassungsrecht 74ff.
}

on stellten. Ganz grundsätzlich ist dazu i.S. einer Kontinuitätswahrung an möglichste Schonung des monarchischen Prinzips zu erinnern. War es im vorausgegangenen Deutschen Bund normativ fixiert (Art. 57 WSA), wird es bis 1918, wenn auch mit fallender Tendenz, gewissermaßen Grundkonstante der BRV bleiben - mit fraglos auch kompetenzieller Relevanz: deshalb der monarchisch beschickte Bundesrat, deshalb - in Abweichung von der amerikanischen Verfassung und mit Nachwirkungen bis heute - der grundsätzliche Verbleib der Ausführung von Reichsgesetzen bei den Einzelstaaten, das bündische System eines Kontingentheeres, der Verzicht auf eine letztentscheidende Verfassungsgerichtsbarkeit und das Absehen von Grundrechten des Reiches zugunsten solcher der Einzelstaaten..$^{25}$ Die BRV sollte sich mithin bis zu ihrem Ende niemals von ihrer doppelfunktionellen Deutung als verfassungsrechtliches Organisationsstatut einerseits und als staatsvertraglicher Fürstenbund andererseits frei machen können. ${ }^{26}$

Für die Throninhaber kamen noch höchstpersönliche Gegebenheiten wie Familienstand, -ehre und -traditionen ins Spiel, die die damals noch virulente Monarchen-Souveränität wie auch Rangstufungen innerhalb der Mitmonarchen berührten. Kompetenzabgaben zugunsten einer Gesamtstaatsbildung auf einvernehmlichem Wege verlangen hier im besonderen Maße diplomatische Finesse, Fingerspitzengefühl und

\footnotetext{
${ }^{25}$ Ausgenommen als Reichsverwaltungsbereiche waren das Post-, Marine- und Konsulatswesen (Art. 48, 53, 56 BRV); zu weiteren optionalen Möglichkeiten HUBER, Verfassungsgeschichte 2 961f. Zum Kontingentwesen, das den Deutschen Bund gekennzeichnet hatte und in den Novemberverträgen von 1870 für Bayern, Sachsen und Württemberg aufrechterhalten wurde, siehe Art. 63f. BRV und ebd. 992 f. Statt eines Verfassungsgerichts wurde die Austrägalgerichtsbarkeit des Deutschen Bundes (Art. XI Abs. 4 DBA) in modifizierter Form durch Art. 76 BRV fortgeführt. ${ }^{26}$ Dazu MeYer, ANSCHÜTZ, Lehrbuch 695ff. mit Fn. s sowie KAUFMANN, Bismarcks Erbe 172ff. und jüngst die Arbeit von HAARDT, Bismarcks ewiger Bund 109ff.
} 
Geschick. So wurde zur Wahrung persönlicher Würde und Ansehen der Monarchen ihre militärische Spitzenstellung jedenfalls als „chef en titre $^{\text {" beibehalten }}{ }^{27}$ und dementsprechend von einer Kompetenz zur Vergabe von Reichsorden abgesehen. Auch blieben überkommene monarchische Steuer- und Portofreiheit unberührt. ${ }^{28}$ Wie peinlich bedacht Bismarck auf einschlägige Rücksichtnahmen war, darüber gibt die meisterliche, wenngleich vielleicht etwas panegyrische Geschichte der Verfassungsentstehung von 1867/71 durch den Neuhistoriker Otto Becker erschöpfende Auskunft. ${ }^{29}$

Als Beispiele für entsprechende Mimikry und Beschwichtigungen Bismarcks seien nur Folgende erwähnt: Erst nach dem Beitritt der kleindeutschen Südstaaten wurde der Name „Deutscher Bund" verfassungsrechtlich durch "Deutsches Reich" ersetzt. ${ }^{30}$ Betont sei weiter die Schonung monarchisch-einzelstaatlicher Empfindlichkeiten dadurch, dass die BRV zur Absicherung einzelstaatlicher Monarchenstellung keinen Regelungsabschnitt über die Regierung, geschweige denn zu Ministern kennt. Als Zentralbehörde wird der unter monarchischer Ägide einzelstaatlich beschickte Bundesrat suggeriert. ${ }^{31}$ Überdies wird die Stimmverteilung der Deutschen Bundesakte von 1815 unter gleicher

\footnotetext{
${ }^{27}$ Dazu Art. 66 BRV.

${ }^{28}$ Zur Steuerfreiheit siehe indirekt schon Art. XIV lit. b DBA; zur Portofreiheit näher KÜHNE, Entstehung 588f. mit Fn. 7.

${ }^{29}$ BECKER, Bismarcks Ringen 290ff.; siehe auch später 525ff. im beschwörenden Brief v. 6.1.1888 an den nachmaligen Kaiser Wilhelm II., die deutschen Mitfürsten gewissenhaft als Bundesgenossen zu betrachten und nicht als Untertanen, die zu parieren hätten.

${ }^{30}$ So noch in Z. I des Bundesvertrags betreffend den Beitritt Bayerns zur Verfassung des Deutschen Bundes vom 23. 11. 1870, HuBER, Dokumente 2, Nr. 220, 200.

${ }^{31}$ In der BRV wird das Wort „Regierung“ nicht einmal verbalisiert, wobei materiell Art. 15 einschlägig ist; zum Bundesrat als gedachter Zentralbehörde s.a. BECKER, Bismarcks Ringen 240.
}

Artikelbezifferung im Wesentlichen übernommen. ${ }^{32}$ Dies bedeutet, dass Preußen gegenüber seinem Bevölkerungsanteil von zwei Drittel im kleindeutschen Raum sein Stimmgewicht um mehr als die Hälfte reduzierte. Seine hegemoniale Dominanz sicherte es sich dann freilich über den etwas versteckten Stichentscheid und die Vetobefugnis bei Verfassungsänderungen sowie über den faktischen Druck seines wirtschaftlichen und militärischen Gewichts. ${ }^{33}$

Weiter gilt hinsichtlich des Heerwesens Folgendes: Seine den Einzelstaaten entgegenkommende Kontingentlösung ist als verfassungsrechtliche Gesetzeskompetenz trotz materieller Unterstützung durch zusätzliche Militärkonventionen so kompromisshaft gestaltet, dass kein Geringerer als Laband die Annahme eines Reichsheeres ablehnte. ${ }^{34}$

Unmissverständliche Zurückhaltung auch unter Beherzigung der alten Sentenz: pecunia nervus rerum. Bismarck ging es darum, jeden Eingriff in das Steuerrecht der Gliedstaaten zu vermeiden. Einnahmequelle des gesamtstaatlichen Dachverbands waren wie im Zollverein Zölle und Verbrauchsabgaben sowie Überschüsse des Post- und Telegraphenwesens. Darüber hinaus waren wie im Deutschen Bund Matrikularbeiträge der Gliedstaaten vorgesehen. ${ }^{35}$ Zwar kam es im Zuge der Verfassungsberatung im konstituierenden Reichstag zusätzlich zu einer eher subsidiarisch formulierten Reichssteuerkompe-

\footnotetext{
${ }^{32} \mathrm{Vgl}$. Art. VI DBA, abgedruckt in: HUBER, Dokumente 1, Nr. 30, 29 und Art. 6 BRV.

${ }^{33}$ So in Art. 5 Abs. 3 und 78 Abs. 1 BRV; zum faktischen Druck KAUFMANN, Bismarcks Erbe 167f. Zu dessen Apotheose des Bismarckschen Bundesrats siehe die quellenmäßige Grundlagenkritik bei KüHNE, Entstehung 110.

${ }^{34}$ Dazu mit Nachweisen zu Laband HUBER, Verfassungsgeschichte 3, 992ff., der sich freilich gegen Laband stellt.

${ }^{35}$ In der genannten Reihenfolge: Art. 38, 49, 70 Satz 2 BRV; zum Deutschen Bund HUBER, Verfassungsgeschichte 1, 608ff., 774f.
} 
tenz, doch wehrte sich Bismarck in seiner Amtszeit stets erfolgreich dagegen, von dieser Kompetenz Gebrauch zu machen. ${ }^{36}$

Weiter ist schließlich hinsichtlich des diplomatischen Geschicks von Bismarck noch auf eine überraschende, ja fast groteske Fehlanzeige aufmerksam $\mathrm{zu}$ machen. Es ist ebenso für seine Rücksichtnahme auf angestammte MonarchenBefugnisse bezeichnend wie der Verfassungswirklichkeit Hohn sprechend, dass sich in der BRV ganz im Gegensatz zu sonstigen deutschen und wichtigen anderen (Bundes-)Verfassungen keine Reichskompetenz für die „auswärtige Gewalt" findet. ${ }^{37}$ Sie zeigt sich nur bruchstückhaft geregelt und wird dem "Gesamtzusammenhang der Verfassung" entnommen. ${ }^{38}$ Doch gehört dies schon zum folgenden Punkt der Kompetenzausschöpfung.

\section{Zur Verfassungswirklichkeit gesamtstaatlicher Kompetenz- erweiterungen}

\section{Rechtstechnische Vorgehensweisen}

Es ist die Wissenschaft zur BRV, die mit bleibender Wirkung für den deutschsprachigen Rechtsraum die schon in der FRV angelegte Unterscheidung von ausschließlichen und konkurrierenden Gesamtstaatskompetenzen durchdrungen hat. ${ }^{39}$ Mitsamt dem Vorrang für jeden-

\footnotetext{
${ }^{36} \mathrm{Vgl}$. die Steuerkompetenz in Art. 4 Nr. 2 und 70 Satz 2 BRV: „solange ...", ein Passus, der durch Gesetz vom 14.5. 1904 (RGBl. 169) gestrichen wurde, womit der provisorische Charakter der Matrikularbeiträge entfiel, siehe auch LABAND, Reichssteuern 13f.

${ }^{37}$ Vgl. Art. 6 FRV, 78 WRV, 10 Nr. 2 B-VG und Art. 32 GG. Lediglich die amerikanische Verfassung weist eine ähnliche Kompetenzzurückhaltung aus, vgl. dazu LOEWENSTEIN, Verfassungsrecht 92f., 306ff.

38 So HUBER, Verfassungsgeschichte 3, 932.

${ }^{39}$ Siehe bereits $\S \S 6,13,40,45$ FRV; zur BRV nur MEYER, ANSCHÜTZ, Lehrbuch 715f.
}

falls kompetenziell einwandfreie Reichsgesetze ist dies nicht weiter $\mathrm{zu}$ vertiefen. ${ }^{40}$ Auch wird man das Fehlen einer vorgeschalteten generellen Bundesstaatsklausel zugunsten der Vorgabe gliedstaatlichen Zuständigkeitsverbleibs ${ }^{41}$ kaum näher thematisieren müssen. Ein entsprechender Antrag nach dem Vorbild der FRV, die der Zuständigkeit der Gliedstaaten nur ausdrückliche Überweisungen an den Gesamtstaat entzogen wissen wollte, wurde im konstituierenden Reichstag als zu theoretisch abgelehnt. ${ }^{42}$

Damit war der Weg frei für eine Deutung der BRV, die über die Ausdrücklichkeit von Reichszuständigkeiten hinaus in schon bemerkter Übereinstimmung mit der amerikanischen Implied-Power-Interpretation ${ }^{43}$ auch stillschweigende Kompetenzbegründungen bejahte. Der alte Auslegungsgrundsatz ,enumerativ bzw. taxativ gleich limitativ" wurde dadurch mit andauernder Wirkung zulasten der Gliedstaaten abgeschwächt, und zwar mit den bis heute geläufigen interpretativen Ausweitungsfiguren: „Natur der Sache“, "Annexzugehörigkeit" und "Sachzusammenhang". ${ }^{44}$

In der damaligen Verfassungspraxis resultiert daraus ein Rattenkönig ausgeweiterter Reichs-

\footnotetext{
${ }^{40}$ Dazu MeYer, AnschüTZ, Lehrbuch 715, Fn. a sowie WIEDERIN, Bundesrecht und Landesrecht 272f.

${ }^{41}$ Z.B. amerikanische Verfassung Zusatzart. X; §5 FRV; Art. 30, 70 Abs. 1 GG.

${ }^{42}$ Dazu näherer Hinweis bei TRIEPEL, Kompetenz 282f.; Antragsvorbild war § 6 FRV, der seinerseits auf Betreiben eines der österreichischen Mitglieder im Verfassungsausschuss der Paulskirche (Eugen Megerle von Mühlfeld) zustande gekommen war, siehe DROYSEN, Verhandlungen 1, 348 (27.9. 1848), und damit Gesamtstaatskompetenzen restringieren wollte - entsprechend einer frühen amerikanischen Auffassung; dazu näher TRIEPEL, Kompetenz 254f. Interessant ist, dass das Ausdrücklichkeitserfordernis im österreichischen Staatsrecht weiterleben sollte; dazu die Beiträge von Simon und Staudigl-Ciechowicz in diesem Band.

${ }^{43}$ Dazu ebd. 256ff. und LoewensteIn, Verfassungsrecht 74ff.

${ }^{44}$ Dazu Triepel, Kompetenz $287 \mathrm{ff}$.
} 
kompetenzen. Dabei lässt sich ihr quantitativer Umfang am besten dadurch veranschaulichen, dass sich Heinrich Triepe ${ }^{45}$ als einer der damals führenden Staatsrechtslehrer in einer knapp hundertseitigen einschlägigen Abhandlung auBerstande sah, sie erschöpfend aufzuzählen. Doch lautet sein Fazit auf dem Stand von 1908, zugleich die amerikanische Verfassung einbeziehend, dahin, dass die geschriebenen Verfassungen der beiden großen Bundesstaaten bei weitem nicht alle Kompetenzen erkennen ließen, mit denen der Gesamtstaat in praxi ausgestattet sei. 46

Kommt hinzu, dass über die vorgenannte Trias ausweitender Interpretationsfiguren hinaus damals noch Erweiterungen durch verfassungsberichtigende Auslegung und Gewohnheitsrecht genannt werden. ${ }^{47}$ Auch diese Ausweitungen gelten als verfassungsmäßig. Dabei wird alsbald und zusätzlich noch das Verfahren der sog. Verfassungsdurchbrechung genutzt und im Zuge der Zeit als gewohnheitsrechtlich legitimiert bezeichnet. ${ }^{48}$ Soll heißen: Ein Gesetz, das sich nicht ausdrücklich als verfassungsändernd bezeichnete, konnte von der Verfassung modifizierend bzw. für den Einzelfall oder generell abweichen. ${ }^{49}$ Nach Art. 78 Abs. 1 BRV, der kein zwin-

\footnotetext{
${ }^{45}$ Ebd. 294.

${ }^{46}$ TRIEPEL, Kompetenz 252.

${ }^{47}$ MeYer, ANSCHÜTZ, Lehrbuch 690; TRIEPEL, Kompetenz 286.

${ }^{48}$ Dazu LABAND, Staatsrecht 39ff., MEYER, ANSCHÜTZ, Lehrbuch 689f. samt dem Beispiel in Fn. 3b des Jesuitengesetzes v. 4.7.1872 (RGBl. 253). Es hatte zwar die Reichskompetenz des Art. 4 Nr. 16 BRV für sich, widerstritt aber ebenso wie das spätere Sozialistengesetz v. 21.10.1878/RGBl. 351) materiell einzelstaatlichen Freiheitsverbürgungen der Religions- $u$. Vereinsfreiheit und überdies der rechtsstaatlichen BRVFundierung, aufgrund deren Ausnahmegesetze an sich ausschieden. Deshalb gingen beide Gesetze nur mit der Annahme impliziter Notwehr- bzw. Notstandskompetenz durch. Zeitgenössische Belege bei KüHNE, Paulskirche 407 Fn. 206.

${ }^{49}$ LABAND, Staatsrecht 38ff.
}

gendes Intextierungsgebot vorsah, durfte einem solchen Gesetz lediglich nicht die dort geregelte Sperrminorität von 14 Stimmen im Bundesrat entgegengesetzt werden. Im Regelfall war es also - wie in England - mit einfacher Mehrheit beschließbar, die notabene auch für - vorbehaltlich des 14-Stimmen-Vetos - intextierte Verfassungsänderungen galt und das Verfahren der Verfassungsdurchbrechung fraglos erleichterte.

Dieses Durchbrechungsverfahren findet sich als Kompetenzüberschreitung zugunsten des Gesamtstaats keineswegs nur bei untergeordneten Materien wie Details des Reichspostgesetzes (1871) oder dem Gesetz zur Reblausbekämpfung (1875). Anzuführen sind vielmehr wie bereits im vorausgegangenen Norddeutschen Bund gravierende Fälle: etwa zu Beginn der 1870er-Jahre zur staatsgebietlichen Einbeziehung Elsaß-Lothringens und in den 1880er-Jahren bei Grundlegung der Bismarckschen Sozialgesetzgebung. ${ }^{50}$ Dass dies keine Einzelfälle waren, dazu sei gleich noch näher auf zwischenzeitliche Eingriffe in die verfassungsnormierten Gubernativstrukturen sowie die föderale Finanzverfassung eingegangen, zu denen es zwischenzeitlich gekommen war.

Als Zwischenergebnis ist jedoch schon hier zu sehen, dass die Reichstagsmehrheit, falls ihr nicht die bundesrätliche Mehrheit oder Sperrminorität entgegengesetzt wurde, in der Kompetenzausweitung denkbar frei war. Insoweit mag man hinsichtlich der Wahl zwischen den Varianten der verfassungsändernden und -durchbrechenden Kompetenzausweitung von einem Glasperlenspiel sprechen. Denn eine verfassungsgerichtliche Kontrolle fehlte. Die damalige Praxis und Doktrin stellte nicht auf die materielle Wichtigkeit eines Rechtssatzes samt dessen Verfassungsrelevanz ab, sondern darauf, ob die Gesetzesvorlage das formale Merkmal der Titelansage einer ausdrücklichen „Abänderung der

\footnotetext{
${ }^{50}$ Zum Ganzen ebd. 41.
} 
Reichsverfassung" enthielt.51 Wann man dieses Merkmal wählte, war jedoch schon laut zeitgenössischem Urteil eines hochkompetenten Staatsrechtslehrers völlig undurchsichtig. ${ }^{52} \mathrm{Im}$ Ergebnis blieb die Wahl mithin dem politischen Ermessen überlassen.

Dies bedeutete im damals echten zweikammerigen Legislativsystem, dass es dem Bundesrat letztlich fakultativ in die Hand gegeben war, seine Sperrminorität gem. Art. 78 Abs. 1 BRV zu aktivieren oder nicht. So lässt sich zwar bedauern, dass unter der BRV in Deutschland anders als in Österreich ein staatsgerichtlich begabtes Reichsgericht als Kompetenzgerichtshof fehlte. Doch hätte dies vermutlich ebenso wenig wie in der Rechtsprechung des amerikanischen Obersten Gerichtshofs strengen Halt geboten. ${ }^{53}$

Bleibt noch zu sagen, dass es die damalige Parlamentspraxis wie überwiegend auch Wissenschaft ablehnte, bei späterer Änderung eines verfassungsdurchbrechenden Gesetzes dem erschwerenden Minoritätsveto des Art. 78 Abs. 1 BRV Raum zu geben. Man ließ dazu vielmehr die einfache Mehrheit genügen, selbst wenn es dabei um noch „weitergehende Modifikationen der ursprünglichen Verfassungssätze“ ging. ${ }^{54}$ Frei nach der Devise: „Beim Ersten bist du frei, beim Zweiten bist du Knecht", wurde damit jedenfalls i.S. von Rechtsklarheit verhindert, dass sich beide Änderungswege miteinander vermengten.

\footnotetext{
${ }^{51}$ Z.B. Gesetz zur Abänderung der Reichsverfassung vom 28. 10. 1918 (RGBl. 1274); s.a. HUBER, Dokumente 3, Nr. 206, 350.

${ }^{52}$ KaUfmanN, Bismarcks Erbe 170.

${ }^{53}$ Dazu Art. 2 StGG-ERG vom 21. 12. 1867 (RGBl. 143/1867); s.a. REITER, Texte Nr. 43, wonach seine Entscheidungsbefugnis freilich nur administrative und nicht auch legislative Kompetenzen betraf, vgl. z.B. ERG I, Nr. 16, 22, 30 usw. Zur amerikanischen Rechtsprechung des Obersten Gerichtshofs herausragend seinerzeit TRIEPEL, Kompetenz 254ff.; s.a. LOEWENSTEIN, Verfassungsrecht 75ff.

${ }^{54}$ Siehe LABAND, Staatsrecht 41.
}

\section{Kompetenzausweitung durch schwachen Verfassungsschutz}

Insgesamt sollte Erich Kaufmann ${ }^{55}$ kurz vor dem faktischen Ende der BRV feststellen, dass die BRV in Bezug auf ihre gesamtstaatliche Legislativausstattung die Schweiz, aber auch die Vereinigten Staaten weit hinter sich lasse. Grund für die vielfältigen Wege zur Kompetenzerweiterung ist ein reduzierter Verfassungsschutz, aufgrund dessen schon zeitgenössisch im Kontrast zur Starrheit der amerikanischen Verfassung die BRV als beweglich bezeichnet worden ist. ${ }^{56} \mathrm{Er}$ schwerend für ihre Änderung ist lediglich das in Art. 78 geregelte, freilich politisch optionale 14Stimmen-Veto im Bundesrat. Ob darüber hinaus die Kompetenz-Kompetenz bestand, im Wege der Verfassungsänderung Umgestaltungen welcher Art auch immer vorzunehmen, d.h. auch im antiföderal-unitarischen und antimonarchisch-republikanischen Sinne, wurde gewissermaßen als Radikalauffassung zwar von Georg Jellinek, Laband und Anschütz vertreten, blieb aber bis 1918 strittig,57 was zu vertiefen sein wird.

Zuvor ist freilich angesichts grundsätzlicher Quorengleichheit für einfache Gesetze wie Verfassungsänderungen noch konkreter die Frage zu stellen: quis iudicabit? Wer entschied damals darüber, ob ein Reichsgesetz die verfassungsgebotenen Kompetenzvorgaben einhielt bzw. gegebenenfalls eine Verfassungsänderung, sei es förmlich, sei es qua Durchbrechung, verlangte?

Die fehlende Verfassungsgerichtsbarkeit bedeutete, dass es hinsichtlich des justiziellen Verfassungsschutzes weder ein konzentriertes, noch ein diffuses richterliches Prüfungsrecht gab. Solche Frage sei der Nachprüfung durch den Richter entzogen, wie es in einer frühen Entschei-

\footnotetext{
${ }^{55}$ KaUfManN, Bismarcks Erbe 192.

${ }^{56} \mathrm{Vgl}$. TRIEPEL, Kompetenz 278.

${ }^{57}$ Eingehend dazu MEYER, ANSCHÜTZ, Lehrbuch 691ff., insbes. m.w.N. 695, Fn. s.
} 
dung des Leipziger Reichsgerichts mit andauernder Wirkung hieß.58 Und hinsichtlich des Verfassungsschutzes durch die Exekutive gab es zwar die Reichsaufsicht ${ }^{59}$ gegenüber Kompetenzüberschreitungen der Gliedstaaten. Bei solchen des Reiches waren es indessen der Reichstag oder Bundesrat, die sich querstellen konnten, aber nicht mussten.

Dies führt zugleich zum legislativen Verfassungsschutz. Hinsichtlich dessen war der Bundesrat anfangs der Auffassung, er allein sei Wächter der Verfassung, womit er dem Reichstag ein entsprechendes Prüfungsrecht absprechen zu können meinte. Doch ließ sich dieser Alleinstellungsanspruch in praxi nicht durchhalten, da die staatspolitische Stellung und Bedeutung des Reichstags auch insoweit mehr und mehr anwuchs. ${ }^{60}$ Freilich ist gleichzeitig in kompetenzieller Hinsicht hinzuzufügen, dass der Reichstag gegenüber der monarchisch-bundesstaatlichen Verfassungsausrichtung mehrheitlich entschieden unitarischer dachte. Insofern war er in der Ausweitung von Reichskompetenzen großzügig, jedenfalls nicht päpstlicher als der Papst, wenn der Bundesrat als Föderativorgan im Zweikammersystem mitzog.

Dies gilt etwa bei der Einrichtung von insgesamt 14 Reichsämtern, zu der es nacheinander bis 1918 weitestgehend im Erlasswege kam. ${ }^{61}$ Sie stieß kaum auf bundesrätlichen Widerstand, ${ }^{62}$

\footnotetext{
58 So RGZ 9, 235f. 1883 st. Rspr.; ebenso. § 30 des Gesetzes betreffend die Organisation des (österreichischen) Reichsgerichts ..., RGBl. 44/1869), s.a. REITER, Texte Nr. 48.

${ }^{59}$ Dazu grundlegend TRIEPEL, Reichsaufsicht, sowie die dogmatische Verfeinerung durch FroweIN, Bundesaufsicht $40 \mathrm{ff}$.

${ }^{60}$ Näher zu Debattenbeispielen im Reichstag HATSCHEK, Staatsrecht 68ff.

${ }^{61}$ Näher HuBER, Verfassungsgeschichte 3, 833ff. und DERS., Dokumente 3, Nr. 251, 393.

${ }^{62}$ So aber hinsichtlich eines Reichsfinanzamtes die Abgeordneten Hänel, VRT 47, 324 (14. Sitz., 5. 3. 1878) und Windthorst, ebd. 340, weiter 1880 hinsichtlich eines Reichsamts für Handel und Gewerbe, siehe HU-
}

obgleich dadurch die Regierungsmimikry der BRV zunehmend zulasten gliedstaatlicher Kompetenzen offensichtlich wird. Und der Reichstag sollte sich mit dem Hinweis auf seine etatgesetzliche Mitsprache beruhigen. ${ }^{63}$

Immerhin wurde im Zusammenhang damit 1878 im Reichstag die Verfassungsmäßigkeit der Kompetenzerweiterung erörtert, nämlich bei Beratung der Regierungsvorlage des Stellvertretergesetzes, das Quasiminister ${ }^{64}$ ermöglichte. Dadurch sollte der Reichskanzler über den klaren Wortlaut von Art. 17 BRV hinaus auch durch die Chefs der Reichsämter vertretbar sein, womit man eine Art Unterverantwortlichkeit schuf. An sich wurde hiermit die gem. Art. 15 BRV alleinige Regierungsverantwortung des Kanzlers i.S. fortschreitender Kollegialisierung und Ertüchtigung der Reichsleitung verändert. Doch gelang es Bismarck erfolgreich, im Bundesrat die gliedstaatlichen Bevollmächtigten darauf einzuschwören, dass erst ein Eingehen auf liberale Forderungen nach Reichsministern ein nicht hinnehmbarer Verfassungsverstoß sei.65 Erst dadurch würde die Balance der verfassungsfixierten, monarchisch-föderalen Waagschalen von Reich und Gliedstaaten beseitigt. Übereinstimmend damit verwiesen hochrangige Stimmführer Bayerns, Württembergs und Sachsens darauf, erst gegenüber solchen Bestrebungen ihre gemeinsame Sperrminorität von 14 Stimmen geltend machen zu wollen. ${ }^{66}$

BER, Verfassungsgeschichte 3, 834. Später sollte es allerdings $\mathrm{zu}$ einem kompetenziell abgespeckten Reichsschatzamt (1879) und einem Reichswirtschaftsamt (1917) kommen, siehe ebd. 833ff.

${ }^{63}$ So die Abgeordneten Hänel, VRT 47, 325 (14. Sitz., 5.3. 1878) und Windthorst, ebd. 432 (17. Sitz., 9. 3. 1878).

${ }^{64}$ Siehe die Abgeordneten Windthorst, VRT 340 (14. Sitz., 5.3. 1878) und Reichensperger, ebd. 393 (16. Sitz., 8. 3. 1878).

${ }^{65}$ Bismarck, VRT 47, 343f. (14. Sitz., 5. 3. 1878).

${ }^{66}$ So die bayerischen, württembergischen und sächsischen Bevollmächtigten: Ministerpräsident A. von Pfretzschner, ebd. 327, Ministerpräsident $H$. von 
Bedenken namhafter Reichstagsabgeordneter, dass die Regierungsvorlage doch die Schaffung von Quasiministern bedeute und man damit den Keim zur Kompetenzausdehnung des Reiches lege, blieben ohne Mehrheit. Diese beruhigte sich vielmehr damit, dass niemand beabsichtige, die Exekutivbefugnisse des Reichs zu erweitern. ${ }^{67}$ Wenngleich es dann faktisch, z.B. durch jedes neue Reichsamt, doch peu à peu dazu kam, wurde insoweit von einer förmlichen Verfassungsänderung 68 doch stets abgesehen dies, obwohl der eine Stellvertretung auslösende klassische Verhinderungsfall in der Praxis alsbald von der Ausnahme zur Regel mutieren sollte. Er wurde nämlich ebenso auf große Geschäftslast wie auf den Mangel technischer Ressortkompetenz erstreckt. Im Klartext lag also eine weitere Verfassungsdurchbrechung vor. ${ }^{69}$

Umgekehrt sollte es ein Jahr später auch zur Verfassungsdurchbrechung zulasten des Reiches kommen. Da seine Zolleinnahmen mit dem antiliberalen Wechsel Bismarcks zum Schutzzoll erheblich stiegen, hätte diese Realentwicklung entgegen der anfänglich austarierten Finanzverteilung unter der BRV dazu geführt, das Reich von den Gliedstaaten finanziell unabhängig zu machen. Um dies zu verhindern, also i.S. bündischföderalen Bestandserhalts, wurden die Reichseinnahmen zugunsten der Gliedstaaten durch die sog. Franckensteinsche Klausel gedeckelt, und zwar in evidenter Abweichung von Art. 38 und $70 \mathrm{BRV} .70$

Mittnacht, ebd. 336f. und Minister H. von NostitzWallwitz, ebd. 391 (16. Sitz., 8. 3. 1878).

${ }^{67}$ So der national-liberale Parteiführer und Abgeordnete R. von Bennigsen, VRT 47, 335 (14. Sitz., 5. 3. 1878); mit anderem, eher psychologisierendem Ansatz, aber gleichem Ergebnis KAUFMANN, Bismarcks Erbe 182 mit Fn. 103.

68 Später so ganz dezidiert der liberale Abgeordnet G. Beseler, VRT 54, 2130f. (77. Sitz., 9. 7. 1879).

${ }^{69}$ Dazu näher HuBER, Verfassungsgeschichte 3, 823f.

70 So näher ebd. 951 m.w.N.

\section{Schlussbewertung}

Insgesamt ist festzustellen, dass die Mimikry gesamtstaatlicher Kompetenzzuweisungen im BRV-Text zwar offiziell aufrechterhalten blieb, mittels vieler Verfassungsdurchbrechungen aber materiell erodiert bzw. unterlaufen wurde. Angesichts solch zwiespältiger Rechtslage kann es nicht verwundern, dass der Entwurf einer Europäischen Verfassung von 198371, der sich kompetenziell an die BRV anlehnte und unter maßgeblicher Beteiligung deutscher EVP-Abgeordneter einschließlich eines Bismarck-Verwandten eingebracht worden war, keine Folge fand. ${ }^{72}$

Andererseits gilt freilich auch, dass der bereits erwähnten, damals prominent vertretenen Lehre i.S. völlig unbegrenzter Verfassungsänderungsbefugnis $^{73}$ in der Praxis durchaus nicht entsprochen worden ist. Vielmehr fällt bei allen Erosionen des gliedstaatlichen Kompetenzbereichs auf, dass sie in dem Maße zurückhaltender werden wie sie der dortigen eigentlichen Monarchenstellung näher rücken. Hier wird gleichsam als Wesensgehalt eine organschaftliche Tabuzone greifbar, die monarchisch-gliedstaatliche Kompetenzträger als solche de iure unangetastet zeigt. Dies ist auch dort zu verzeichnen, wo faktischer Monarchenentfall eine partiell unitarisierende Neugliederung nahegelegt hätte. Insoweit erweisen sich die in Art. 6 BRV aufgeführten Gliedstaaten samt ihrer ponderierten Stimmenzahl bis 1918 als sakrosankt.

Dazu sei in aller Kürze auf den Umgang mit seinerzeitigen Thronvakanzen mangels Thronerben verwiesen, was an sich eine Staatenzusammenlegung eröffnet hätte. Das gilt etwa für Braunschweig, dessen Thronvakanz ab 1884

\footnotetext{
${ }^{71}$ Entwurf zur Ausarbeitung einer Europäischen Verfassung vom 26.9.1983, in SCHWARZE, BIEBER, Verfassung für Europa 571ff.

72 Vgl. Art. 4 BRV und 48 Entwurf, ebd., sowie Art. 70 BRV und 70f.; bei dem Bismarck-Verwandten handelte es sich um Philipp von Bismarck (1913-2006). ${ }^{73}$ S. o. III 2 mit Fn. 57.
} 
reichsaufsichtlich knapp 30 Jahre offengehalten wurde. ${ }^{74}$ Und es gilt ebenso für die beiden Lippe, wo es ab 1895 im Wege der bundesrätlichen Austrägalkompetenz in Art. 76 BRV erst nach 10 Jahren zur trennungswahrenden Neubesetzung des verwaisten Detmolder Throns kam. ${ }^{75}$ Anders wiederum in den thüringischen Duodezfürstentümern der beiden Reuß und beiden Schwarzburg: Hier rettete man ab 1909 die jeweilige Zweistaatlichkeit ähnlich Sachsen-Coburg-Gothas durch Personalunionen. ${ }^{76}$ Lediglich im Fall der beiden Mecklenburg wurde interterritorial noch 1918 die Vereinigung der beiden Landesteile Mecklenburg-Schwerin und Mecklenburg-Strelitz vereinbart. Doch sollte dem endgültigen Vollzug ausgerechnet die Novemberrevolution, und zwar um einen Tag zuvorkommen ${ }^{77}$ - ein Zufall, der es bei der dortigen Zweistaatlichkeit mit Wirkung für Weimar beließ. Dies war durchaus nicht nur kleinstaatliche Posse. Über den Untergang der BRV hinaus stand es vielmehr für breitere föderale Beharrung, die nochmals kontrapunktisch das Gewicht der überkommenen deutschen Föderalstrukturen samt damit verbundener Kompetenzen bewies.

Entgegen der bereits erwähnten These radikaler Veränderbarkeit ${ }^{78}$ sprach die Praxis bis 1918 mit Nachwirkungen auf die anschließende WRV durchaus für die weitverbreitete Mindermeinung, die neben Seydel als dem bayrischen Verfechter entschieden bündischer Reichssicht unter Vorantritt der Staatsrechtler Georg Meyer, Otto Mayer, Erich Kaufmann und Erwin Jacobi

\footnotetext{
${ }^{74}$ Dazu Huber, Verfassungsgeschichte 4, 428ff., TRIEPEL, Reichsaufsicht 458f. mit Fn. 2 und FroweIN, Bundesaufsicht $53 f$.

${ }^{75}$ HUBER, Verfassungsgeschichte 4, 433ff.

${ }^{76} \mathrm{Zu}$ Reuß und Schwarzburg siehe ebd. 1058, Fn. 41 und 216, Fn. 46.

77 Dazu genau ebd. 427 f.; zur dabei evtl. notwendigen Änderung von Art. 6 BRV siehe MEYER, ANSCHÜTZ, Lehrbuch 696, Fn. s.

${ }^{78}$ S. o. Pkt. III 2 mit Fn. 57.
}

i. S. einer Art Ewigkeitsgarantie die bundesstaatlichen Grundlagen einer Verfassungsänderung nach Art. 78 BRV entzogen sah bzw. für ein Abgehen davon Einstimmigkeit im Bundesrat verlangte. ${ }^{79}$

Dem entsprach, dass alle Überlegungen zu einer bloßen Partialrevision der BRV, die der spätere Stammvater der WRV Hugo Preuß noch 1917 in Umlauf gebracht hatte, nach der Novemberrevolution ein Jahr später chancenlos waren. Dasselbe galt übrigens auch im Blick auf eine Totalrevision in Form des damals kurzzeitig virulenten Gedankens, nun die FRV - freilich monarchisch purifiziert - in Kraft zu setzen. Obwohl der damals führende Neuhistoriker Friedrich Meinec$\mathrm{ke}^{80}$ eine Wiederanknüpfung an die großdeutschen Hoffnungen der Achtundvierziger ebenso positiv sah wie an deren Farben, riet er davon ab. Für ihn war diese Verfassung auch für die Gliedstaaten zu sehr monarchisch geprägt.

Genauer ließe sich dazu ihre Ablehnung der Volkssouveränität zugunsten einer nationalsouveränen Zweieinheitlichkeit von Fürst und Volk anführen. ${ }^{81}$ Die Abschaffung solcher Parität auch de iure war nach der faktischen Beseitigung durch die primär sozial-revolutionär geprägte Novemberrevolution von 1918 ohne Werbekraft. Und nicht zuletzt war angesichts der anfangs massiven gebietlichen wie kompetenziell unitarisierenden Neugliederungsbestrebungen nicht zu übersehen, dass diese Verfassung nunmehr hinter aktuellen bundesstaatlichen Bedürfnissen des Reiches zurückblieb. ${ }^{82}$ Dass der ursprüngliche Neugliederungselan dann trotz der zur WRV

\footnotetext{
79 Näher MEYER, ANSCHÜTZ, Lehrbuch 693, Fn. m sowie KAUFMANN, Bismarcks Erbe 150 und 172f. hinsichtlich der vertraglichen Grundlagen und speziell des Art. 78 Abs. 2 BRV; für Einstimmigkeit JACOBI, S. $98 \mathrm{ff}$. Bis auf Kaufmann wirkten die Genannten im Wesentlichen an nichtpreußischen Universitäten.

${ }^{80}$ Dazu näher HoLBORN, Verfassung 118f.

${ }^{81}$ Dazu eingehend KÜHNE, Entstehung 572ff.

${ }^{82}$ Holborn, Verfassung $116 \mathrm{f}$.
} 
führenden Totalrevision dem Schattenwurf der BRV Tribut zu zollen hatte, verweist bereits auf den folgenden Beitrag.

\section{Korrespondenz:}

Prof. Dr. Jörg-Detlef KüHNE

Leibniz Universität Hannover

Emeritierter Professor

Königsworther Platz 1

D-30167 Hannover

kuehne@jura.uni-hannover.de

ORCID-Nr. 0000-0002-6342-3313

\section{Abkürzungen:}

BRV Bismarcksche Reichsverfassung

ERG Erkenntnisse des österreichischen RG, d.h. Anton Hye von GlunECK (Hg.), Sammlung der nach gepflogener öffentlicher Verhandlung geschöpften Erkenntnisse des k.k. österreichischen Reichsgerichtes, Bd. 1 (Wien 1874).

FRV Frankfurter Reichsverfassung

i.S. im Sinne

RGZ Entscheidungen des Reichsgerichts in Zivilsachen

s.o. siehe oben

st. Rspr. ständige Rechtsprechung

s.u. siehe unten

Sitz. Sitzung

VRT Verhandlungen des Reichstages

WSA Wiener Schlussakte

Siehe auch das allgemeine Abkürzungsverzeichnis: [http://www.rechtsgeschichte.at/media/abk.pdf]

\section{Literatur:}

Otto BECKER, Bismarcks Ringen um Deutschlands Gestaltung (Heidelberg 1958).

Otto v. BISMARCK, Gedanken und Erinnerungen (Stuttgart 1965).

Michael DREYER, Der Föderalismus als ordnungspolitisches und normatives Prinzip. Das föderale Denken der Deutschen im 19. Jahrhundert (Frankfurt a.M. 1987).

Johann Gustav Droysen (Hg.), Die Verhandlungen des Verfassungs-Ausschusses der deutschen Nationalversammlung, Teil 1 (Leipzig 1849).
Jochen FrowEIN, Die selbständige Bundesaufsicht nach dem Grundgesetz (Bonn 1961).

Otto GIERKE, Labands Staatsrecht und die deutsche Rechtswissenschaft, in: Jahrbuch für Gesetzgebung, Verwaltung und Volkswirtschaft im Deutschen Reich 7 (1883, ND Darmstadt 1961) 10981195

Oliver HAARDT, Bismarcks ewiger Bund (Darmstadt 2020).

Julius HATSCHEK, Deutsches und Preußisches Staatsrecht, Bd. 1 (Berlin 1922).

Hajo HolBorN, Verfassung und Verwaltung der dt. Republik. Ein Verfassungsentwurf Friedrich Meineckes aus dem Jahre 1918, in: HZ 147 (1933) 115128.

Franz von HOLTZENDORFF, Ernst BezOLD, Materialien der Deutschen Reichs-Verfassung. Sammlung sämtlicher auf die Reichs-Verfassung, ihre Entstehung und Geltung bezüglichen Urkunden und Verhandlungen einschließlich insbesondere derjenigen des constituierenden Norddeutschen Reichstags 1867, Bd.1-3 sowie alphabetisches Sprech- und Sachregister (Berlin 1873; ND 1976).

Ernst Rudolf HuBER (Hg.), Dokumente zur deutschen Verfassungsgeschichte, Bd. 1-3 (Stuttgart-BerlinKöln $\left.{ }^{3} 1978-1990\right)$.

Ernst Rudolf HUBER, Deutsche Verfassungsgeschichte seit 1789, Bd. 1-4, (Stuttgart u.a. ${ }^{21975-1994), ~ B d . ~} 5$ (ebd. $\left.{ }^{1} 1980\right)$.

Ludolf HuGO, Zur Rechtsstellung der Gebietsherrschaften in Deutschland, dt. Übersetzung der 1661 erschienenen Helmstedter Dissertationsschrift „De statu regionum Germaniae" durch Yvonne PfaNNENSCHMID (Münster 2005).

Erwin JACOBI, Der Rechtsbestand der deutschen Bundesstaaten (Leipzig 1917)

Erich KAUFMANN, Bismarcks Erbe in der Reichsverfassung (Berlin 1917, ND in: DERS., Gesammelte Schriften, Bd. 1, Göttingen 1960) 143-223.

Jörg-Detlef KÜHNE, Die Reichsverfassung der Paulskirche. Vorbild und Verwirklichung im späteren deutschen Rechtsleben (Neuwied 21998).

DERS., Die Entstehung der Weimarer Reichsverfassung. Grundlagen und anfängliche Geltung (Düsseldorf 2018).

Paul LABAND, Direkte Reichssteuern. Ein Beitrag zum Staatsrecht des Deutschen Reichs (Berlin 21908).

DERS., Das Staatsrecht des Deutschen Reiches, Bd. 2 (Tübingen 51911, ND Goldbach 1997).

Karl LOEWENSTEIN, Verfassungsrecht und Verfassungspraxis der Vereinigten Staaten (Berlin-Göttingen-Heidelberg 1959). 
Georg MEYER, Gerhard ANSCHÜTZ, Lehrbuch des Deutschen Staatsrechts (München-Leipzig ${ }^{71919) . ~}$

Yvonne PfANNENSCHMID, Ludolf Hugo (1632-1704). Früher Bundesstaatstheoretiker und kurhannoverscher Staatsmann (Baden-Baden 2005).

William Emanuel RAPPARD, Die Bundesverfassung der Schweizerischen Eidgenossenschaft 1848-1948 (Zürich 1948).

Ilse REITER (Hg.), Texte zur österreichischen Verfassungsentwicklung (Wien 1997).

Martin P. SCHENNACH, Kompetenzverteilung avant la lettre? Methodisch-theoretische und wissenschaftshistorische Reflexionen zur Zuständig- keitsverteilung in vormodernen Mehrebenensystemen, in: BRGÖ 11 (2021) 61-99.

Jürgen Schwarze, Roland BIEBER (Hg.), Eine Verfassung für Europa (Baden-Baden 1984).

Heinrich TRIEPEL, Die Kompetenz des Bundesstaats und die geschriebene Verfassung, in: Staatsrechtliche Abhandlungen, Festgabe Paul Laband, Bd. 2 (Tübingen 1908; ND Frankfurt a.M. 1978) 249-335.

DERS., Die Reichsaufsicht (Berlin 1917).

Ewald WIEDERIN, Bundesrecht und Landesrecht. Zugleich ein Beitrag zu Strukturproblemen der bundesstaatlichen Kompetenzverteilung in Österreich und in Deutschland (Wien-New York 1995). 